\title{
Inflammatory response of the spinal cord to multiple episodes of blood-brain barrier disruption and toxic demyelination in Wistar rats
}

C.G. Fernandes ${ }^{1}$,

D.L. Graça ${ }^{2}$ and

L.A.V.D. Pereira ${ }^{3}$

\author{
1Departamento de Patologia Animal, Faculdade de Veterinária, \\ Universidade Federal de Pelotas, Pelotas, RS, Brasil \\ 2Departamento de Patologia, CCS, Universidade Federal de Santa Maria, \\ Santa Maria, RS, Brasil \\ ${ }^{3}$ Departamento de Histologia/Embriologia, Universidade de Campinas, \\ Campinas, SP, Brasil
}

\section{Correspondence \\ C.G. Fernandes \\ Departamento de Patologia Animal \\ Faculdade de Veterinária, UFPel \\ 96010-900 Pelotas, RS \\ Brasil \\ Fax: 55 (0532) 75-9004 \\ E-mail: crisgf@fmb.unesp.br}

Research supported by CNPq

(No. 402092/89/9). Publication

supported by FAPESP.

Received March 12, 1997 Accepted January 15, 1998

\section{Abstract}

Multiple episodes of blood-brain barrier disruption were induced by sequential intraspinal injections of ethidium bromide. In addition to the barrier disruption, there was toxic demyelination and exposure of myelin components to the immune system. Twenty-seven 3-monthold Wistar rats received 2, 3 or 4 injections of $1 \mu$ of either $0.1 \%$ ethidium bromide in normal saline (19 rats) or $0.9 \%$ saline ( 8 rats) at different levels of the spinal cord. The time intervals between the injections ranged from 28 to 42 days. Ten days after the last injection, all rats were perfused with $2.5 \%$ glutaraldehyde. The spinal sections were evaluated macroscopically and by light and transmission electron microscopy. All the lesions demonstrated a mononuclear phagocytic infiltrate apparently removing myelin. Lymphocytes were not conspicuous and were found in only $34 \%$ of the lesions. No perivascular cuffings were detected. In older lesions (38 days and older) they were found only within Virchow-Robin spaces. This result suggests that multiple blood-brain barrier disruptions with demyelination and exposure of myelin components to the immune system were not sufficient to induce an immune-mediated reaction in the central nervous system.

At present, there is no convincing explanation for the immune reactions against myelin that occur in different demyelinating diseases, such as distemper in dogs and multiple sclerosis in humans $(1,2)$. Such an immune response is characterized basically by lymphocytes, mononuclear perivascular cuffings and demyelination $(3,4)$. In several studies of toxically induced demyelinating processes, no lymphocytes were detected, but they were found in the ethidium bromide

\section{Key words}

- Lymphocytes

- Multiple sclerosis

- Central nervous system

- Blood-brain barrier

- Ethidium bromide
(EB) model in the spinal cord of Wistar rats. This was attributed to blood-brain barrier disruption and the general inflammatory reaction that followed (5). After multiple injections of lysophosphatidyl choline into the sciatic nerves, lymphocytes were also found, and interpreted to be part of an immune response (6).

In the present study we investigate the occurrence of an immune-mediated process after multiple episodes of blood-brain bar- 
rier disruption and demyelination induced by EB in Wistar rats and compare the morphological aspects observed with those of other spontaneous $(1,2)$ or experimental $(7)$ immune-mediated demyelinating processes in men and animals.

Twenty-seven 3-month-old Wistar rats of both sexes (4 females and 23 males) were submitted to 2, 3 or 4 intraspinal injections of $1 \mu \mathrm{l}$ of $0.1 \%$ EB saline solution (19 rats) or $0.9 \%$ saline (8 rats) into alternate locations between the 8 th thoracic vertebra and the 4 th lumbar vertebra. The injections were performed at intervals of $28,32,35$ or 42 days.

For the injections, the rats were anesthetized with fentanyl/hypnomidate (Johnson \& Johnson, São José dos Campos, SP, Brazil) and a burrhole was drilled in the dorsal spinous process of the target vertebra. The injections (EB or saline) were performed by hand, using a Hamilton syringe. The wound was sutured and the animals were allowed to recover. The animals were killed 10 days after the last injection by intracardiac perfusion with $2.5 \%$ glutaraldehyde and $2 \%$ paraformaldehyde in $0.1 \%$ phosphate buffer, $\mathrm{pH}$ 7.2-7.4. The injected area was identified and 1-2-mm transverse sections were cut, postfixed with osmium tetroxide after washing

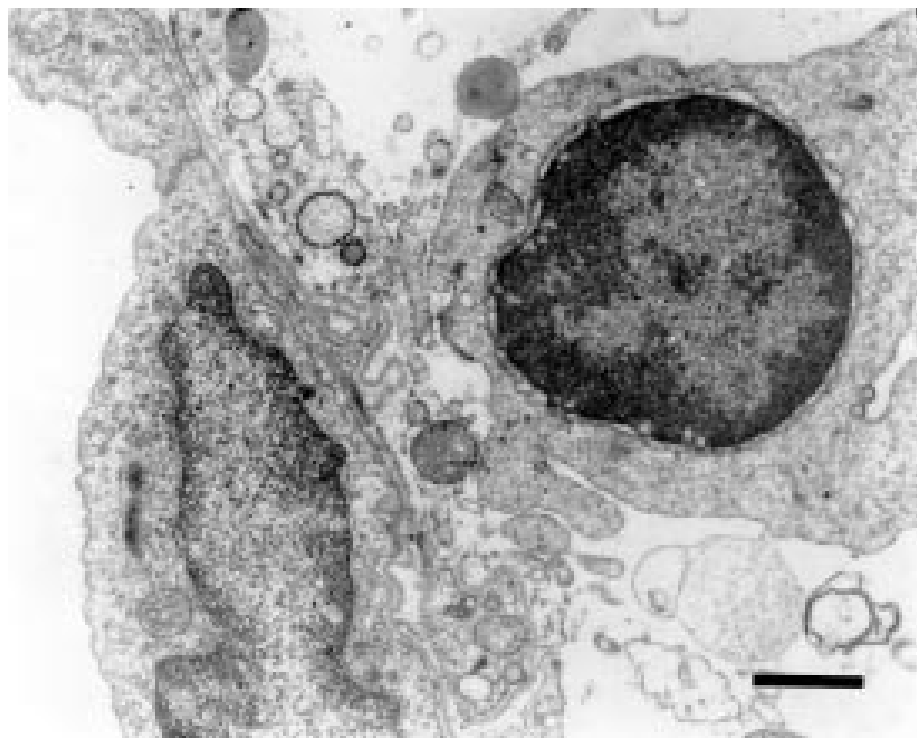

Figure 1 - Lymphocyte within a Virchow-Robin space. Bar $=1 \mu \mathrm{m}$. with $2 \%$ glutaraldehyde in sodium cacodylate buffer $(0.03 \mathrm{M}, \mathrm{pH} 7.3)$, dehydrated with upgraded concentrations of ethanol, and embedded in Epoxy 812 resin. Semithin sections $(1 \mu \mathrm{m})$ were cut and stained with methylene blue/blue II for light microscopy. Selected areas were trimmed and ultrathin sections were stained with uranyl and lead salts and examined with a Zeiss EM 10 transmission electron microscope operated at $60 \mathrm{kV}$.

Primary demyelination was induced by EB following glial cell death, with preservation of vascular structures. The lesions varied in size and histological appearance and could later be remyelinated predominantly by Schwann cells, chiefly in subpial and perivascular areas, or by oligodendrocytes in areas close to normal tissue. In some samples remyelination did not occur, even in old lesions (136 days postinjection) where hyperplasia and hypertrophy of astrocytes and persistence of myelin debris in the extracellular space were the predominant features.

In all lesions, macrophages were recognized by their round nuclei and abundant cytoplasm with phagocytosed particles such as lipid droplets and myelin debris. Ultrastructurally they revealed clearer nuclear densities and shapes than lymphocytes and a distended cytoplasm with numerous phagosomes delimited by membranes. They were more abundant in the center of the early lesions, between demyelinated axons and cellular debris. Macrophages were also found in perivascular areas, but were less activated.

Lymphocytes were recognized by light microscopy by their small hyperchromatic nuclei and scanty cytoplasm. The study of these cells on serial semithin sections was indispensable to differentiate them from macrophages or Schwann cells, mainly in perivascular locations. By electron microscopy lymphocytes were recognized by their dark and small nuclei with condensed chromatin (Figure 1). Occasionally they had a small cytoplasm where polyribosomes, scarce mitochondria, granular endoplasmic reticu- 
lum and small Golgi complexes were detected. Lymphocytes were found in only 18 of 53 samples (34\%), usually in subpial or perivascular areas around lesion boundaries and uncommonly within the lesions. Within perivascular spaces lymphocytes lay between the basal laminae of the glia limitans and endothelial cells or pericytes, and never invaded the neuropil. They appeared singly and never in clusters or forming perivascular cuffings. They did not have close relationships with macrophages or other cell types.

Changes induced by $0.9 \%$ saline injection were initially characterized by intraand extracellular swelling (10 days postinjection). After 38 days post-injection, there were also features of Wallerian degeneration along the needle track. A few macrophages infiltrated the area and no changes in glial cells were observed.

Mononuclear phagocytes were the predominant cells and were more abundant in the center of the lesions. Similar features were reported in EB-induced lesions in the brain $(8,9)$ and after one intraspinal injection (10). Fast activation of mononuclear phagocytes is characteristic of primary demyelination. It is likely that the attracting signal for macrophage entry is specific and transient (11). This would explain the persistence of myelin debris within the extracellular space of some lesions, when adequate macrophage activation did not take place after oligodendrocyte death. In such situations myelin loses its lipid components and only the protein moiety persists which is not at all attractive to mononuclear phagocytes (12). In addition to the breakdown of myelin sheaths by myelinolytic enzymes, macrophages also perform phagocytosis of cellular debris and myelin (13) and act in CNS reparative processes. They can release cellular factors such as the angiogenic molecules transforming growth factor $\beta_{1}$ and interleukin-1, the latter also being an inducer of astrocyte proliferation. Macrophages also induce collagen fiber release which may be the proliferative and migratory stimulus for the invading Schwann cells (10).

It is likely that after sequential EB injections, the entry of inflammatory cells was facilitated by the lack of an active bloodbrain barrier. The blood-brain barrier disruption occurred after the injection trauma unavoidable in this sort of model (14), and also following astrocyte destruction since astrocytes can be responsible for the expression and maintenance of blood-brain barrier (15). Since lymphocytes and plasma cell infiltrates were not a consistent finding in these lesions, it seems reasonable to propose that in this model there is no immune-mediated demyelinating feature. Additionally, no mononuclear perivascular cuffings, which are related to persistent antigenic expression (16) characteristic of chronic relapsing demyelinating processes $(1,2,7)$, were found. Relapsing demyelination, another feature of these immune-mediated diseases, was not observed in this study. The lymphocytes observed in the model of a single EB injection (5) were probably part of the general inflammatory response to the nonspecific effects of EB. It seems possible that the scant lymphocytic infiltrate is the same as observed in acute inflammations of the CNS, when there is blood-brain barrier disruption and the cells can easily gain access to nervous tissue (4). In older lesions, where the glia limitans was restored, a few lymphocytes occur only within the Virchow-Robin spaces, where they can be found even in normal situations (16).

The findings suggest that no exposure of an antigenic myelin fragment that could induce an immune demyelinating response occurred in EB-induced lesions, although the antigenic potential of some myelin components, such as myelin basic protein, has been demonstrated $(2,17)$.

Thus, it is likely that many factors, besides blood-brain barrier disruption, are involved in immune-mediated demyelinating processes in the CNS, such as multiple sclerosis, including autoimmune factors (18) such 
as Thelper (Th) lymphocytes specific against myelin basic protein and activation of astrocytes, endothelial cells or microglia (17). Environmental and genetic factors could also be involved, as well as a viral agent (19), that may incite a cross-reaction against myelin basic protein (17) or actually cause the disease $(17,18,20)$. Some investigators $(20)$ suggest that the etiology of multiple sclerosis is multifactorial, and that sclerosis only occurs when some of these factors coincide in the same individual.

\section{References}

1. Braund KG, Brever BD \& Mayhew IG (1987). Inflammatory, infectious, immune, parasitic and vascular diseases. In: Oliver Jr JE, Hoerlein BF \& Mayhew IG (Editors), Veterinary Neurology. W.B. Saunders, London, 216-254.

2. Raine CS (1990). Multiple sclerosis. Immunopathologic mechanisms in the progression and resolution of inflammatory demyelination. In: Waksman BH (Editor), Immunologic Mechanisms in Neurologic and Psychiatric Disease. Raven Press, New York, 37-54.

3. Blakemore WF (1982). Myelination, demyelination and remyelination in the CNS. In: Cavanagh JB \& Smith WT (Editors), Recent Advances in Neuropathology. Churchill Livingstone, Edinburgh, 53-81.

4. Leibowitz $S$ (1983). The immunology of multiple sclerosis. In: Hallpike JF, Adams CWM \& Tourtellote WW (Editors), Multiple Sclerosis. Pathology, Diagnosis and Management. Chapman and Hall, London, 379-412.

5. Graça DL (1988). The presence of lymphocytes in a toxically induced demyelinating process of the central nervous system. Microscopia Electronica y Biologia Celular, 12: 17-22.

6. Hall SM (1984). The effects of multiple sequential episodes of demyelination in the sciatic nerve of the mouse. Neuropathology and Applied Neurobiology, 10: 461-478.
7. Raine CS (1983). Multiple sclerosis and chronic relapsing EAE: comparative ultrastructural neuropathology. In: Hallpike JF, Adams CWM \& Tourtellote WW (Editors), Multiple Sclerosis. Pathology, Diagnosis and Management. Chapman and Hall, London, 413-458.

8. Yajima K \& Suzuki K (1979). Ultrastructural changes of oligodendroglia and myelin sheaths induced by ethidium bromide. Neuropathology and Applied Neurobiology, 5: 49-62.

9. Pereira LAVD (1994). Efeitos do brometo de etídio no tronco cerebral de ratos Wistar: aspectos morfológicos relacionados aos processos de desmielinização e remielinização do sistema nervoso central. Master's thesis, UNICAMP.

10. Graça DL \& Blakemore WF (1986). Delayed remyelination in rat spinal cord following ethidium bromide injection. Neuropathology and Applied Neurobiology, 12: 593-605.

11. Beuche W \& Friede RL (1984). The role of non-resident cells in wallerian degeneration. Journal of Neurocytology, 13: 767796.

12. Offner H \& Konat G (1983). Myelin changes induced by incubation of brain slices with serum. Neurochemistry International, 5: 45-49.

13. Cammer W, Brosnan CF, Bloom BR \& Norton WT (1981). Degradation of the $P_{0}$, $\mathrm{P}_{1}$ and $\mathrm{P}_{\mathrm{r}}$ proteins in peripheral nervous system myelin by plasmin: implications regarding the role of macrophages in demyelinating disease. Journal of Neurochemistry, 36: 1506-1514.

14. Ozawa K, Saida T, Saida K, Nishitani H \& Kameyama M (1989). In vivo CNS demyelination mediated by antigalactocerebroside antibody. Acta Neuropathologica, 77: 621-628.

15. Risau W (1991). Induction of blood-brain barrier endothelial cell differentiation. Annals of the New York Academy of Sciences, 633: 405-419.

16. Prineas JW (1979). Multiple sclerosis: presence of lymphatic capillaries and lymphoid tissue in the brain and spinal cord. Science, 203: 1123-1125.

17. Deber CM \& Reynolds SJ (1991). Central nervous system myelin: structure, function and pathology. Clinical Biochemistry, 24: 113-134.

18. Tourtellotte WW \& Pick PW (1989). Current concepts about multiple sclerosis. Mayo Clinics Proceedings, 64: 592-596.

19. Selmaj K, Raine CS, Farooq M, Norton WT \& Brosnan CF (1991). Cytokine cytotoxicity against oligodendrocyte - apoptosis induced by a lymphotoxin. Journal of Immunology, 147: 1522-1529.

20. Haar S, Mette S \& Møller-Larsen A (1991). Multiple sclerosis as a retroviral disease? Epidemiological considerations in relation to HTLV-1 epidemiology. Neuroepidemiology, 10: 232-235. 